\title{
Digital planning and guided surgery in dental implants: a case report
}

\author{
L. Zanettini, P. Zanettini, W. Polido, R. Pagnoncelli
}

Background: Dental implants are constantly evolving, with several new technologies. Nowadays, it is the most sought after treatment option for individuals who are candidates for oral rehabilitation, improving esthetics and recovering masticatory capacity. Always in search of minimally invasive procedures, dentistry has sought ways to decrease surgical time, decrease morbidity and greater safety of the surgical procedure. Based on these searches digital planning and guided surgery are being widely used, with the possibility of greater predictability and precision in the planning of dental implants, giving greater chances of successful surgery along with the technique performed correctly.

Objectives: The present study aims to report a clinical case, in which digital planning and guided surgery for oral rehabilitation with dental implants were used.

Methods: A 27-year-old female patient attended the Private Clinic with functional and aesthetic complaint. At the intra-oral clinical examination, facial analysis and computed tomography, the patient had missing tooth13 and 23 , which were previously removed because they were impacted and without the possibility of orthodontic traction. Dental implants rehabilitation was the recommended treatment alternative for this case.The surgical planning consisted of upper and lower arch digital scanning, digital planning in coDiagnostiXTM (Dental Wings Inc.) software, preparation of the prototyped surgical guide printed on a 3D printer.

This is the author's manuscript of the work published in final form as:

Zanettini, L., Zanettini, P., Polido, W., \& Pagnoncelli, R. (2019). Digital planning and guided surgery in dental implants: A case report. International Journal of Oral and Maxillofacial Surgery, 48, 209. https://doi.org/10.1016/j.ijom.2019.03.643 
Findings: The surgery was performed under local anesthesia in the clinic. After the installation of the dento-supported prototyped surgical guide (supported on the adjacent teeth), the perforations follow the conventional system protocol (Straumann Guided Surgery). The basic requirements of implant placement surgery, such as good primary stability and the correct position guided by the future restoration, were observed. In the case described, excellent primary stability was achieved, which allowed placement of the implants, and waiting only 6 weeks for osseointegration.

Conclusion: Along with the literature review and the case report, it is shown that digital planning and guided surgery seek greater predictability, morbidity reduction and greater safety of the surgical procedure, giving positive results to the operative practice.

This is the author's manuscript of the work published in final form as:

Zanettini, L., Zanettini, P., Polido, W., \& Pagnoncelli, R. (2019). Digital planning and guided surgery in dental implants: A case report. International Journal of Oral and Maxillofacial Surgery, 48, 209. https://doi.org/10.1016/j.ijom.2019.03.643 